\title{
Qualitative Study of Stakeholder Perceptions Related to Requirements for Elimination of Dog Rabies in Sri Lanka by 2025
}

Kamalika Harchini Ubeyratne Janrabelge ${ }^{1,2 *}$, Lertrak Srikitjakarn ${ }^{2}$, Dirk Udo Pfeiffer ${ }^{3}$, Sunil-Chandra Narapity Pathirannehalage ${ }^{4}$, Lisa Kohnle ${ }^{3}$, Warangkhana Chaisowwong ${ }^{2}$, Phonpat Hemwan ${ }^{5}$

${ }^{1}$ Veterinary Research Institute, Sri Lanka

${ }^{2}$ Faculty of Veterinary Medicine, Thailand

${ }^{3}$ City University of Hong Kong, Hong Kong

${ }^{4}$ University of Kelaniya, Sri Lanka

${ }^{5}$ Faculty of Social Sciences, Thailand

*Corresponding author: Kamalika H.U. Janrabelge: kamalikau@yahoo.com

\section{OPEN ACCESS}

Citation: Janrabelge K.H.U., Srikitjakarn L., Pfeiffer D.U., SunilChandra N.P., Kohnle L., Chaisowwong W., Hemwan P. (2019) Qualitative Study of Stakeholder Perceptions Related to Requirements for Elimination of Dog Rabies in Sri Lanka by 2025. Open Science Journal 4(1)

Received: $16^{\text {th }}$ October 2018

Accepted: $9^{\text {th }}$ May 2019

Published: $17^{\text {th }}$ July 2019

Copyright: (C) 2019 This is an open access article under the terms of the Creative Commons Attribution License, which permits unrestricted use, distribution, and reproduction in any medium, provided the original author and source are credited.

Funding: The author(s) received no specific funding for this work

Competing Interests: The author have declared that no competing interests exists.

\section{Abstract:}

Dog rabies has been successfully eliminated in a number of Asian countries, including Japan, Singapore, the Republic of Maldives, and Hong Kong. In Sri Lanka, human rabies cases declined from 288 in 1975 to 25 in 2018, supporting the country's commitment to global elimination of dog-mediated human rabies by 2030. This positive trend is largely due to the provision of intradermal postexposure prophylaxis, but it has only been one cornerstone to success.

This study discusses stakeholder perceived obstacles to more increased rabies control in the context of strengthening existing strategic approaches, including promotion (surveillance, diagnosis, reporting, vaccination), prevention (responsible dog ownership, dog population management), and partnership development (coordinated approaches) in Sri Lanka. The objective was to identify and evaluate these obstacles in the face of a targeted elimination of dog-mediated human rabies in the country by 2025 . A qualitative data collection was performed between August and December 2017. Nine focus group discussions with dog owners from local communities and seven in-depth interviews with representatives of governmental animal and human health systems, local government, and non-government organizations were conducted. 
Topics comprised dog rabies reporting, dog vaccination coverage, reasons for dog population increases and challenges of the present rabies control system.

Those focus group discussions with community residents and indepth interviews with stakeholders, government officials, and non-government agents revealed that the main obstacles to rabies control perceived by participants included the need for increased motivation to report the disease and the development of a clear protocol for disease reporting by the public. In some locations, a lack of awareness of the importance of disease reporting was found, which was exacerbated by logistical issues, uneven vaccination coverage due to insufficient communication related to government vaccination campaigns, and incomplete implementation of existing government policies. Moreover, an integrated national dog rabies monitoring and reporting system, based on effective partnership among relevant institutions, as well as a larger number of decentralized laboratories for dog rabies diagnosis, including additional local veterinary and medical government staff, are needed. Other issues identified are the need for more responsible dog ownership and an improved understanding of rabies disease risks amongst dog owners. Dogassociated factors that were mentioned include the need to improve the understanding of drivers of variation in dog population size, to reduce the economic costs of sterilization for owners and to monitor post-surgical complications. The weakest link in rabies elimination in Sri Lanka is prevention at the source: current institutional capacity is insufficient to achieve the goal of $70 \%$ dog vaccination coverage.

To eliminate dog-mediated human rabies in Sri Lanka by 2025, a stronger inter- and cross-sectoral collaboration between all stakeholders is needed. This study identified and assessed important obstacles in overcoming known challenges of rabies control and will help to develop effective strategies that can support the country on its way to elimination, if supported by a legal framework.

Keywords: Stakeholders, Dog rabies, Elimination, Qualitative analysis 


\section{Introduction}

Differences exist among countries in implementing dog rabies vaccination campaigns aimed at reaching the World Health Organization (WHO) recommended $70 \%$ coverage with coverage being a key indicator of ability to carry out mass dog vaccination (1). Rabies control programs require intersectoral coordination and communication (2) exemplified in WHO's five rabies elimination pillars (STOP-R): socio-cultural, technical, organizational, political, and resource approaches (3). In each country, local socio-cultural context influences perceptions of rabies and dog-keeping practices, e.g., responsible dog ownership, dog population management, and vaccination. Animal and public health systems have a key role in rabies vaccination coverage, including logistic, diagnostic, surveillance and technical support. Government authorities provide enforcement of legal frameworks. Other partners, including funding agencies, give long-term support (4). In many instances, however, control and prevention initiatives have been insufficient. In developing countries, poor community participation in local rabies control programs together with technical, intersectoral, organizational, and monetary obstacles slow progress toward preventing human rabies through control of the disease in its dog populations (5) even though elimination of dogmediated human rabies is technically feasible through a combination of dog vaccination and post-exposure prophylaxis for humans $(6,7)$. Requirements for successful elimination of dog-mediated rabies include prevention by vaccination of dogs, education of the public, cost-effective and high-quality human rabies vaccines, sufficient local capacity for rabies diagnosis, effective disease reporting and surveillance, and responsible dog ownership (5).

To be successful, national rabies prevention and control requires all stakeholders to work together. Sri Lanka is committed to the goal of global elimination of dog-mediated human rabies worldwide by 2030 (8). Studies in Northern Australia and in Papua New Guinea have identified obstacles to community engagement, e.g., reasons for underreporting sick dogs, insufficient veterinary services, lack of treatment response and a lack of trust in authorities (9). To help develop new strategies and boost the efficacy of efforts to eliminate dog-mediated rabies in Sri Lanka, it is important to identify country-specific factors by exploring the views, perceived needs, and practices of stakeholders. The objective of this study was to evaluate perceived obstacles to achieving dogmediated rabies elimination in Sri Lanka by 2025.

\section{Materials and Methods}

\section{Study area selection}

The five highest number of canine rabies cases in Sri Lanka during 2004 to 2016 occurred in 25 districts. One of those, of the five districts with the highest number of canine rabies cases during 2004 to 2016, Galle district, was selected for study by simple randomization. Out of 19 Divisional Secretariat Divisions, the highest incidence of $\operatorname{dog}$ rabies in Galle, 125 for the period 2004-16, occurred in 
Hikkaduwa Divisional Secretariat Division (DSD), which was purposively selected for this study.

The study area, Boossa Grama Niladhari Division (GND) of Hikkaduwa Divisional Secretariat Division, Galle District (Fig. 1) was selected by simple random sampling from the 97 GNDs in Hikkaduwa DSD. The densely populated majority Buddhist and Sinhalese area has a well-developed infrastructure, good employment opportunities, as well as human and animal health service facilities. The economy is primarily based on natural resource exploitation (10).

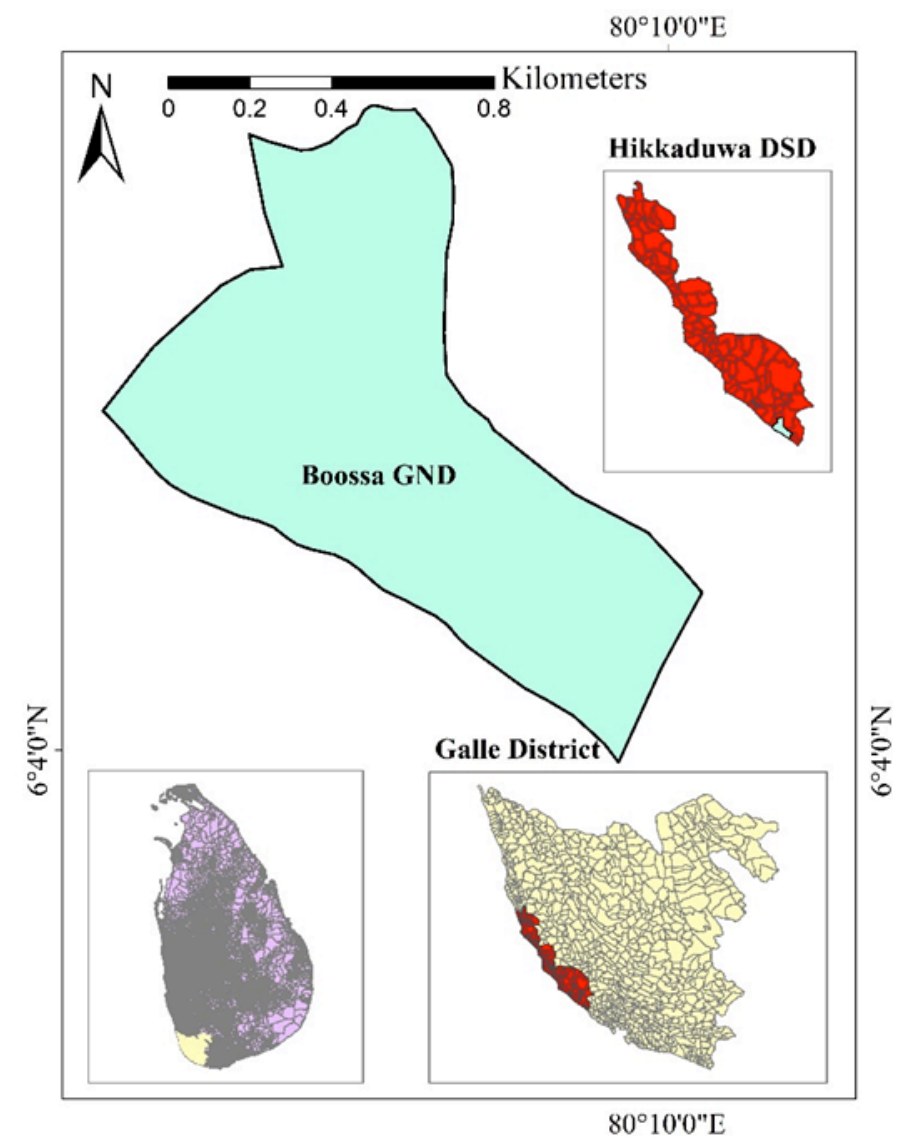

Figure 1. Study site

\section{Data collection}

The study was conducted between August and December 2017, using focus group discussions (FGD) (Table 1) and in-depth, semi-structured interviews. 
Table 1. Focus group discussion themes and sample questions used to identify factors influencing the effectiveness of rabies prevention and control in Boossa GND

FGD themes and sample questions

Theme 1: Levels of dog rabies reporting and identification of risk areas

- How do you report dog rabies to authorities?

- $\quad$ To whom do you report dog rabies?

- How long does it take for owners to report a sick dog with signs of rabies?

Theme 2: Levels of dog vaccination coverage

- What is the level of uptake of anti-rabies vaccine by users?

- What are the reasons for uneven and insufficient vaccination coverage across the country in both domestic and stray dogs?

Theme 3: Reasons for dog population increases

- What factors lead to dog population increases?

For both FGDs and in-depth semi-structured interviews, target groups/participants were selected by purposive sampling. Criteria for FGD participants were dog ownership and equal gender representation. Expertise and involvement in rabies control were used for selecting interviewed participants.

\section{Focus group participants}

The sixty focus group participants represented a range of dog-owners (Table 2) were divided into gender-balanced groups of 6 to 8 , and all gave prior oral consent for recording the discussions. Group discussions were continued until sufficient data had been obtained and no new information was emerging. 
Table 2. Focus group participant characteristics

\begin{tabular}{|c|c|c|l|}
\hline $\begin{array}{c}\text { Focus } \\
\text { group }\end{array}$ & $\begin{array}{c}\text { Number of } \\
\text { participants }\end{array}$ & $\begin{array}{c}\text { Age range } \\
\text { (years })\end{array}$ & \multicolumn{1}{|c|}{ Occupations } \\
\hline 1 & 6 & $55-70$ & Self-employed; housewife; business owner; retiree \\
\hline 2 & 8 & $27-60$ & Housewife; teacher; private sector employee; self-employed; nurse; retiree \\
\hline 3 & 6 & $28-55$ & Housewife; retiree; self-employed; business owner \\
\hline 4 & 6 & $32-68$ & Housewife; retiree; self-employed \\
\hline 5 & 8 & $24-66$ & $\begin{array}{l}\text { Housewife; masonry worker; private sector employee; business owner; } \\
\text { railway employee }\end{array}$ \\
\hline 6 & 6 & $19-70$ & Retiree; business owner; housewife; high school/college student \\
\hline 7 & 6 & $53-78$ & Self-employed; housewife; business owner; retiree \\
\hline 8 & 6 & $45-74$ & Housewife; retiree; fishery sector; port authority employee \\
\hline
\end{tabular}

\section{In-depth semi-structured interviews}

Seven in-depth semi-structured interviews lasting 45 to 60 minutes were conducted with representatives of governmental animal and human health systems, local government, and non-government organizations following Food and Agriculture Organization (FAO) and International Livestock Research Institute (ILRI) guidelines (11) regarding reasons for weaknesses in the dog rabies control system. Interviewees were informed about the topic and gave oral consent to the interview.

Interviews explored obstacles to prioritizing dog rabies, including assignment of responsibilities, budgetary allocation, human and animal health sector coordination, disease surveillance, disease reporting and laboratory diagnosis, implementation of government policies, and obstacles to dog vaccination.

\section{Data analysis}

All interviews were recorded and transcribed. Qualitative data from the FGDs and in-depth interviews were examined, and relevant words or phrases associated with specific research questions were categorized. Quotations in this report are translations from the participants' mother tongue (Sinhala). Information obtained was triangulated using different data gathering methods and different groups for credibility validation. Analysis was performed following FAO and ILRI guidelines (11).

This study received prior approval from the Committee for Ethical Clearance on Animal Research, Faculty of Veterinary Medicine and Animal Science, University of Peradeniya, Sri Lanka (approval code VER-2017-003). 


\section{Results}

Community perceptions of obstacles to rabies prevention and control are described below. Participant comments are in italics.

\section{Obstacles to effectiveness of strategic approaches to promotion of rabies control}

\section{a. Dog rabies reporting and reasons for underreporting}

Community understanding of rabies reporting routes varied. FGD participants said they would report incidents to officers representing government agencies including veterinary services, e.g., veterinary surgeons, livestock development instructors (FG5); public health services, e.g., public health inspectors, midwives (FG3, FG4), and medical officers (FG9); public administration officials, e.g., Grama Niladhari (FG4); or state defense services, e.g., police (FG9). Some participants were not aware to whom they should report an incident or even that they were required by law to report. Typical comments include, "I am not aware that there is a responsible person available" (FG6). Others stated they would not inform officials, but would inform fellow villagers for their protection, e.g., "I would inform people I meet walking along the road" (FG7). Many focus groups (FG9, FG6, FG7, FG2) indicated that reporting is not practiced and that mad dogs are killed and buried by villagers if there are no human dog bite victims.

Six key reasons for gaps in community involvement in rabies reporting were identified. Experience-based decisions, e.g., mistrust of authorities due to prior experiences where people felt they had received an inappropriate response (FG4). Knowledge-based decisions including lack of awareness of the importance of surveillance, e.g., "After killing a mad dog, the problem is solved" (FG6) and inability to recognize disease signs, e.g., "I have no experience, so I cannot recognize a rabid dog" (FG7). Animal care cultural norms including differences in perceived responsibility for stray and owned dogs, e.g., "No one bothers about stray dogs. They have no identity. If they are mad, kill them and bury them" (FG7). Perceptions of risk levels, e.g., "If somebody is bitten by an owned dog, tie up the dog and, if it dies, submit the head' (FG3). Unwillingness to report and lack of risk awareness, e.g., "People don't like to bother and are afraid to cut the head off a rabid dog because of fear of rabies exposure" (FG1). "Now incidents are rare due to vaccination. We only see rabies in stray or free-roaming dogs" (FG3). Logistical obstacles, e.g., "The police station is far away. Rabid dogs move around, so I can't wait. I kill and bury them" (FG2), "I have no way to transport the head' (FG5).

\section{b. Reasons for uneven and insufficient vaccination coverage}

The FGDs identified seven groups of obstacles to vaccination of domestic and stray dogs. Organizational factors. These included insufficient communication about health programs and government vaccination campaigns, campaigns conducted on an irregular schedule or on workdays; campaigns held at short notice; low level of dedication among control programs officers; insufficient public 
awareness about vaccination campaigns, e.g., "We receive only short notice of campaigns. People are not available on workdays" (FG5); insufficient time allocated to each center, e.g., "By the time I learned of the campaign and took my dog, the vaccination team had moved on to a new location, so I missed out' (FG3). It was also mentioned that vaccination of stray dogs had not happened for many years, e.g., "No one comes to catch the stray dogs. Earlier, there was such a program, but I have not seen it for 6 or 7 years (FG7); "Operators only go along the main roads but not the by-roads, so vaccination occurs mostly at locations where a high number of dogs are available. There is no post-vaccination monitoring" (FG9). In general, vaccination campaigns are appreciated, e.g., "Not everybody can afford to pay for vaccination, so the campaign is good"; "Now there are less frequent reports of the disease due to vaccination" (FG2). Communitybased factors. Most people have affection for their dogs and take them to be vaccinated, but some irresponsible owners say, e.g., "I have no time; I am busy with other work" (FG6). Some refrain from participating when the vaccination point is not at a community reserved place (FG2). Another factor was lack of responsible ownership, e.g., "I only provide food, not vaccines"; "I am unwilling to invest my time or money. I only provide food but not shelter (FG3). Distance to the vaccination point and inability of government agencies to serve the periphery. "People are unwilling to take their dogs to the vaccination point or to the veterinary office without a vehicle. If a mobile service is available, we would prefer that". "The temple is too far away. When vaccination was conducted at a nearby house, we vaccinated". Knowledge- and attitude-based decisions. Some community elders and vaccinators erroneously believe the vaccine is too strong for pups and that dogs confined at home are safe from the disease. Unvaccinated dogs are viewed as commonplace, e.g., "My female dog had given birth to pups, so I thought it was not good to have her vaccinated." (FG9). Dog-associated factors. These included difficulties taking dogs to the vaccination center, dog aggressiveness and unwillingness of to be put on a leash (FG5). "My dog was afraid when it saw the other dogs, broke its leash and ran away" (FG6). Social pressure from the community. "If our dog bites a neighbor, they will inquire about the vaccination record, so we vaccinate, but some others pretend the dog does not belong to them and do not vaccinate". Failure to implement government policies. Some government regulations are implemented poorly and do not effectively motivate individuals to have their dogs vaccinated (FG8).

\section{Obstacles to effective implementation of strategic prevention}

\section{a. Factors influencing dog population increases}

The first population management theme was economic obstacles and community cultural attitudes. Free-roaming dogs belonging to low income families are seen as common, e.g., "Some people in the community cannot afford to provide shelter or vaccinations; they only provide leftover food, so responsibility for providing veterinary care often falls to government and nongovernment organizations" (FG1). Stray dogs are the major source of dog populations; however, negative attitudes toward birth control were also noted, 
e.g., "Reduction in the numbers of local breeds of dogs due to birth control has been observed" (FG3); "The natural behavior of domestic dogs should not be interfered with" (FG6). Religious concerns associated with birth control were also mentioned, e.g., "We believe that surgical measures are a sin" (FG6). Comments on a second theme, animal care culture, included, "Stray dogs in the community were initially household dogs, but lack of responsible pet ownership combined with roaming in areas where household dogs meet community dogs creates packs of dogs which live in the street" (FG5); "Mobility of household dogs is high during the mating season" (FG2). Participants mentioned abandoning unwanted pups (especially females) at temples, abandoned houses, open lands, along roadsides, or in cemeteries. Several comments referred to feeding stray dogs and open garbage dumps, both of which facilitate survival of strays, e.g., "Out of sympathy, when we have excess food, we provide food for roaming dogs". It was felt that stray dogs in a village provide protection, e.g., "Thieves are scared when stray dogs are roaming in the night' (FG2). Another theme related to insufficient dog population management and post-sterilization operation complications. Participants thought stray dogs were not included in animal birth control programs, e.g., "I have not seen stray dogs being caught and sterilized for 6 or 7 years" (FG8). "Following sterilization surgery some dogs suffered from infection along the surgical line." (FG6); "There is no pain management provided following the procedure, so the animal suffers" (FG5). Several comments referred to the distance to the sterilization point and government staff's unwillingness to serve peripheral locations, e.g., "If they come to a nearby place we will take our dogs, otherwise we will not go". Participants also mentioned knowledge-based factors, e.g., "People are not aware of the gravity of rabies" (FG5); "Only knowledgeable people get their dogs sterilized" (FG7). Several comments referred to social changes such as human population increases, urbanization, rising social standards, and lack of safety from thieves (FG3) as leading to an increase in the domestic dog population.

\section{In-depth interviews}

Perspectives of stakeholders belonging to the animal, human health systems, local governments and non-government organizations were explored with respect to their experience with long-term rabies control, especially obstacles negatively impacting promotion of rabies control and hindering inter- and intra-institute partnerships.

\section{Obstacles affecting effective implementation of strategic approaches to promotion of rabies control}

\section{a. Disease surveillance, reporting, and laboratory diagnosis}

Animal health system interviewees mentioned lack of high-quality surveillance data on rabies in dogs, e.g., "Dog rabies cases are underreported and the level of reporting is not monitored. There are insufficient diagnostic facilities and no infrastructure for dog rabies diagnosis". Non-governmental organization (NGO) 
members mentioned, "There is no established grassroots level reporting system. Lack of monitoring of dog rabies reporting and lack of dog rabies data hinder identification of high risk areas." Local government interviewees made statements such as, "There is no active surveillance. Laboratory capacity is low. The general public has not been provided a clear protocol for disease reporting"; "There is only one Public Health Inspector for each area, so monitoring and coordination is not optimal. Infrastructure for disease reporting has not been established". Interviewees from the public health system said, e.g., "Only human rabies surveillance has been performed adequately. There is no island-wide animal rabies surveillance"; "For cultural reasons as well people do not remove the heads of dogs for submission"; "If a dog bites people, they get vaccinated and do not report'.

\section{b. Problems facing mass dog vaccination}

Interviewees recognized the challenge of achieving vaccination coverage above 70\%. Animal health system participants noted remaining obstacles, e.g., "No accurate dog rabies surveillance data to identify risk zones for strategic vaccination, so every population must be vaccinated above $70 \%$ plus emergency protection when there are reports of human cases in a local area. Ensuring sufficient vaccine availability will require additional funds. Dog turnover and dog population size data is necessary to predict vaccine needs. Ensuring the safety of vaccinators is a problem. Due to dog turnover, herd immunity is not maintained'. Those from NGOs said, e.g., "There is no baseline data on the dog populations, domestic or stray, to determine vaccine needs". Local government interviewees said, e.g., "National vaccination coverage is less than $70 \%$ due to a lack of institutional capacity. Financial regulations preclude purchasing quality vaccines. Vaccine failures are not reported. Regulations requiring vaccination campaigns is a must". Animal health system staff made comments such as, "Not enough staff is available, distribution of vaccines is not monitored, vaccination is not evenly distributed or regular, and there is no post-vaccination monitoring". Public health system interviewees added, "30\% of dogs submitted as suspected rabies cases had been vaccinated. Even some vaccinated dogs have become rabies positive after booster vaccinations. This suggests vaccination failures that have not been identified due to lack of monitoring"; "During field vaccinations, cold chain maintenance is not monitored"; "Vaccinators are careless when administering vaccines to animals."

\section{Obstacles to successful implementation of strategic approaches related to partnerships}

\section{a. Prioritizing dog rabies}

Animal health system interviewees mentioned, "Island-wide distributed laboratories for animal rabies diagnosis are lacking. Dog rabies surveillance data are deficient, masking the extent of dog rabies. Livestock deaths due to rabies are not recorded. There is insufficient evidence to accurately describe the dog rabies 
situation."; "Dog rabies is not reported by the general public; information on dog bites is not shared with veterinary surgeons; dog bites are reported only to the public health system; the number of facilities for dog rabies diagnosis is low." NGOs mentioned, e.g., "The animal health sector does not allocate funds for dog rabies control, funding goes to the human health sector instead. PEP masks the dog rabies problem." One local government interviewee said, "There are no decentralized rabies diagnosis laboratories, so dog rabies positives are underreported. Public health system statements included, e.g., "People's awareness regarding dog rabies is low."

\section{b. Responsibility allocation among stakeholders}

Animal health system comments included, "Authority for dog rabies control is held by local governments". An NGO representative said, "The current rabies ordinance needs revision to fit today's situation; responsibilities must be clearly identified." A local government staffer said, "There should be a centralized monitoring system under the supervision of the Department of Animal Production and Health for technical activities related to rabies control and animal rabies surveillance by local governments." Public health system representatives mentioned, e.g., "If a human rabies death is reported, a ring vaccination within a $5 \mathrm{~km}$ radius for all dogs is needed, but that is not being done."; "Rural local government authorities consider rabies control a public health issue, not something in which they are required to participate."

\section{b. Budget allocation}

An animal health system interviewee said, "Policy makers need to be convinced of the need for funding by demonstrating the impact of rabies. The animal production and health system allocates much attention to livestock diseases, but attention to dog rabies is low." Public health system staff mentioned, "Local governments do not have a separate rabies prevention budget. Rabies prevention is not given priority." Non-government organization staff said, "Budget is unevenly allocated among the animal health and human health systems, with more going to the human health system." Local government staff said, "Sterilization impact is low, so spending on that is a waste. The labor cost of vaccination is low compared to the high cost of sterilization."

\section{c. Coordination between human health and animal health}

Animal health system interviewees indicated, "There is no national platform linking organizations, no data sharing between the human and animal health systems." An NGO staff member said, "Local governments do not have enough technical capacity; the animal health system lacks sufficient infrastructure and trained manpower for rabies diagnosis; elimination of rabies requires intersystem coordination." A local government employee stated, "Intra-agency coordination does not exist, so inter-agency cooperation is unlikely." Public health system interviewees said there is no scientific knowledge sharing "especially about dog vaccination coverage and the status of the rabies control process. No real-time data is available. There is no forum for stakeholders"; 
"Government hospital out-patient departments handle curative aspects of dog bite victims and Medical Officers of Health handle dog rabies control activities, but there is no link between them".

\section{e. Implementation of government policies}

A local government employee said, "Priority areas have not been identified, e.g., dog registration and promotion of responsible pet ownership. There were no resources or institutions to implement the policies at the time the rabies ordinance was enacted. Now the institutions and resources are available, but there is no coordination. Policies should be science-based." One NGO interviewee stated, "The existing policies are sufficient, but are not being implemented." Another animal health system participant mentioned, "The general public lacks awareness of laws and regulations related to dog rabies control. The government has prioritized other areas." Public health system staff said, "There is a system in place, but implementation and monitoring of the system is not happening."

\section{Discussion}

The extent to which rabies elimination priorities are shared by the community and the government strongly influences elimination effort effectiveness. Obstacles emerging as challenges to rabies elimination include limited access to veterinary care, large free-roaming dog populations, and substandard animal welfare practices (12). In northern Australia and Papua New Guinea, there is a lack of veterinary services (9), and in Peru access to dog rabies vaccination is a problem (13).

Dog rabies reporting is essential to prevention of human rabies, facilitates rabies post-exposure prophylaxis and counseling for individuals exposed to rabid animals, helps ensure capture and confinement of rabid animals, and facilitates histological examination of animal brains (14). Although reporting rabies is legally required, gaps in community and organizational response result in underreporting of dog rabies. Field-level respondents in animal health, public health, and public administration indicated they abandoned disease reporting due to lack of experience, the local animal care culture, inaccurate understanding of the risk, and unwillingness to handle dog heads, among other logistical issues.

In many countries, appropriate decisions regarding control efforts cannot be made because passive surveillance of canine rabies has resulted in significant underreporting (15). Reasons identified for underreporting of dog rabies include lack of awareness and motivation and lack of clear protocol for disease reporting by the public, insufficient infrastructure and staff for dog rabies diagnosis, and lack of monitoring of the dog rabies reporting system.

Canine vaccination has been demonstrated to be cost-effective in preventing human rabies and reducing post-exposure prophylaxis expenditures (16). Prevention through mass dog vaccination remains the best option $(17,18)$ but requires at least $70 \%$ coverage (19). Other factors negatively impacting vaccine uptake include lack of responsible dog ownership, inaccurate understanding, e.g., vaccine is too strong for pups, and lack of knowledge of the importance of preexposure immunization. Other areas include insufficient communication with 
communities about vaccination campaigns, distance to vaccination points, and dog aggressiveness.

The level of dog vaccination coverage and the degree of geographical homogeneity within an area affect a country's ability to conduct successful mass dog vaccination campaigns and to eliminate dog rabies (1). Presently, vaccination coverage in Sri Lanka is neither geographically homogenous nor high enough (20).

Factors affecting capacity to achieve a $70 \%$ dog vaccination identified in interviews include absence of monitoring of vaccination failures, inadequate vaccine distribution, lack of post-vaccination investigation, lack of $\mathrm{dog}$ rabies surveillance data to identify high risk zones, lack of capacity to increase motivation of the general public, lack of dog population data to determine vaccine needs, insufficient funds for vaccine, lack of a system to ensure vaccine quality, and no legal obligation for dog owners to vaccinate.

Sustainable high dog immunization coverage depends greatly on dog population management which is the responsibility of rabies control planners. Understanding local dog populations, community attitudes, assessments of dog population management programs, plus less expensive and less labor-intensive dog sterilization tools are needed for effective and sustainable dog population management to eliminate rabies $(21)$.

Animals rabies data is insufficient as few specimens are submitted to government diagnostic laboratories (22). Interview responses indicated this was a reason for the inability to prioritize dog rabies and was due to insufficient decentralized rabies diagnosis laboratories, the use of PEP masking dog rabies, not sharing human dog bite incident information with the animal health system, lack of awareness of notifiable diseases, and lack of disease reporting by the public.

Although local governments have authority for dog rabies control, responsibility is shared with the Ministry of Health due to insufficient local government institutional capacity. In 2012, the Ministry of Livestock and Rural Community Development (LRCD) became a collaborative partner with the Ministry of Health with responsibility for the dog component of rabies control. However, this study suggests successful rabies elimination requires multi-sectoral collaboration and centralized monitoring under the Department of Animal Production and Health.

In general, the failure of current control programs in dog rabies-affected countries can be attributed to insufficient funding compounded by a lack of awareness of the true burden of the disease leading to rabies being perceived as low priority relative to other health programs (5). Although sufficient funds have been allocated to the public health system in Sri Lanka, funding specifically for rabies control in the animal health system is insufficient. Program cost reduction, e.g., lower cost methods for animal birth control are needed.

Successful canine rabies elimination in the Western Hemisphere, Western Europe, and some Asian countries has been achieved through mass dog vaccination, dog population control, and coordination at the national and community levels with support and promotion by national governments (1). During interviews, state level stakeholders (public health, animal health, local governments, and non-government organizations) stressed the need for effective inter-agency coordination. 
Although free-roaming dogs are very unlikely to be counted in a dog census, free-roaming family-owned dogs can be counted through a program of dog registration which could extend vaccination and increase traceability of freeroaming dogs (21). Specific legislation implemented in Singapore (23) and licensing and compulsory dog vaccination in Malaysia (24) were key to achieving freedom from rabies. In the current study, absence of dog registration and lack of responsible pet ownership, lack of mandatory vaccination, lack of specific laws and regulations, and lack of awareness on the part of the general public were among the obstacles identified by stakeholders.

\section{Conclusions}

Although Sri Lanka has successfully reduced human rabies, complete elimination will require increased strategic cooperative efforts by the Public Health Department of the Ministry of Health, the Animal Health Department of the Ministry of LRCD, the Department of Local Government of the Ministry of Provincial Councils and Local Government, and non-government organizations. Evaluation of obstacles faced by local partners in rabies control is a critical first step in developing more effective strategies. Impediments to dog rabies control identified by stakeholders, factors which hamper the country's ability to eliminate dog-mediated rabies, include inadequate efforts to increase geographically homogenous vaccination coverage, limited understanding of local dog rabies epidemiology, logistical constraints, operational challenges, lack of resources, competing priorities for implementation of control activities (mainly dog vaccination), lack of dog population management and dog rabies surveillance, as well as incomplete reporting and diagnosis of rabies. Achieving elimination of dog rabies will require effective strategies, full commitment, coordination, cooperation, and an appropriate legal framework supported by sustainable allocation of funds.

\section{Acknowledgements}

The authors gratefully acknowledge the support of the Skoll Global Threats Fund (grant number 14-02328) to the Participatory OneHealth Disease Detection (PODD) project, Chiang Mai University, Thailand. We would also like to thank the community of the Boossa Grama Niladhari Division for their cooperation, Grama Niladhari of Boossa Grama Niladhari Division for his assistance, the Government Veterinary Surgeon of the Rathgama Government Veterinary Office and the Livestock Development Officers who facilitated the field study, and for Dr. G.L. Robert for editing the manuscript and guiding in qualitative study. 


\section{References:}

1. Wallace RM, Undurraga EA, Blanton JD, Cleaton J, Franka R. Elimination of dog-mediated human rabies deaths by 2030: needs assessment and alternatives for progress based on dog vaccination. Front Vet Sci. 2017 Feb;4(9):1-14.

2. Cleaveland S, Lankester F, Townsend S, Lembo T, Hampson K. Rabies control and elimination: a test case for one health. Vet Rec. 2014 Aug;175(8):188-93.

3. WHO, OIE. Global elimination of dog-mediated human rabies: the time is now! Report of the Rabies Global Conference 10-11 December 2015, Geneva, Switzerland. Paris: World Health Organization and World Organisation for Animal Health. 2016;p.27. [cited 2017 Nov 08]. Available

from: http://apps.who.int/iris/bitstream/10665/204621/1/WHO_HTM_NTD_NZD_2016.02_e ng.pdf? $\mathrm{a}=1$.

4. Fahrion AS, Taylor LH, Torres G, Müller T, Dürr S, Knopf L. et al. The road to dog rabies control and elimination- what keeps us from moving faster?. Front Public Health. 2017 May;5(103):1-8.

5. Aga AM, Hurisa B, Urga K. Current situation of rabies prevention and control in developing countries: Ethiopia perspective. J Infect Dis Preve Med. 2016 Feb;4(1):1-6.

6. WHO. WHO Expert Consultation on Rabies, Second Report. Geneva. World Health Organization. 2013..

7. WHO. Rabies: Rationale for Investing in the Global Elimination of Dog-Mediated Human Rabies. Geneva. World Health Organization. 2015.

8. OIE. OIE members. 2017; [cited 2017 Dec 05]. Available from; http://www.oie.int/about-us/ourmembers/member-countries/.

9. Brookes VJ, Kennedy E, Dhagapan P, Ward MP. Qualitative research to design sustainable community-based surveillance for rabies in Northern Australia and Papua New Guinea. Front Vet Sci. 2017 Feb;4(19):1-9.

10. PRA. Performance Report \& Accounts-2015, District Secretariat-Galle. 2015; [cited 2018 Jul 24]. Available from: http://www.parliament.lk/uploads/documents/paperspresented/performance-report-districtsecretariat-galle-2015.pdf.

11. FAO and ILRI. A manual for practitioners in community-based animal health outreach (CAHO) for highly pathogenic avian influenza. 2010; [cited 2018 Apr 20]. Available from: http://www.fao.org/docrep/014/i1799e/i1799e00.pdf.

12. Schildecker S, Millien M, Blanton JD, Boone J, Emery A, Ludder F. et al. Dog ecology and obstacles to canine rabies control in the Republic of Haiti, 2014-2015. Transbound Emerg Dis. 2017 Oct;64(5):1433-1442.

13. Castillo-Neyra R, Brown J, Borrini K, Arevalo C, Levy MZ, Buttenheim A. et al. Barriers to dog rabies vaccination during an urban rabies outbreak: qualitative findings from Arequipa, Peru. PLOS Negl Trop Dis. 2017 Mar;11(3):1-12.

14. WSDH. Rabies suspected exposure. Washington State Department of Health. 2017 Sep; 1-22. [updated $2017 \quad$ Sep; cited $2017 \quad$ Sep 12]. Available from: https://www.doh.wa.gov/Portals/1/Documents/5100/420-073-Guideline RabiesSuspectedExposure.pdf.

15. Taylor LH, Hampson K, Fahrion A, Abela-Ridder B, Nel LH. Difficulties in estimating the human burden of canine rabies. Acta Trop. 2017 Jan;165:133-40.

16. Fitzpatrick MC, Hampson K, Cleaveland S, Mzimbiri I, Lankester F, Lembo T et al. Costeffectiveness of canine vaccination to prevent human rabies in rural Tanzania. Ann Intern Med. 2014 Jan;160(2):91-100.

17. Knobel DL, Lembo T, Morters M, Townsend SE, Cleaveland S, Hampson K. Dog Rabies and Its Control. In: Jackson AC, editor. Rabies. 3rd ed. Elsevier Inc;. 2013. p. 591-615.

18. Hampson K, Coudeville L, Lembo T, Sambo M, Kieffer A, Attlan M. et al. Estimating the global burden of endemic canine rabies. PLOS Negl Trop Dis. 2015 Apr; 9(4):1-20.

19. WHO. WHO expert consultation on rabies. WHO Technical Report Series 931. First report. Geneva. World Health Organization. 2005; p.1-88.

20. Harischandra PAL, Gunesekera A, Navaratnasingam J, Gongal G, Abela-Ridder B. Sri Lanka takes action towards a target of zero rabies death by 2020, WHO South-East Asia J Public Health. 2016 Sep;5(2):113-16. 
21. Taylor LH, Wallace RM, Balaram D, Lindenmayer JM, Eckery DC, Mutonono-Watkiss B. et al. The role of dog population management in rabies elimination- A review of current approaches and future opportunities. Front Vet Sci. 2017 Jul;4(109):1-15.

22. Karunanayake D, Matsumoto T, Wimalaratne O, Nanayakkara S, Perera D, Nishizono A. et al. Twelve Years of Rabies Surveillance in Sri Lanka, 1999-2010. PLOS Negl Trop Dis. 2014 Oct;8(10):1-14.

23. Association of Southeast Asian Nations (ASEAN). ASEAN Rabies Elimination Strategy; OIE World Organisation for Animal Health: Bangkok, Thailand. 2016 Dec; [cite 2018 Apr 23]. Available from: http://asean.org/storage/2017/02/ASEAN-Rabies-EliminationStrategy.pdf.

24. Bamaiyi PH. 2015 outbreak of canine rabies in Malaysia: review, analysis and Perspectives. J Vet Adv. 2015 Dec;5(12):1181-1190. 